\title{
Entre modèles nationaux et eurogroupes d'intérêts professionnels : l'action de l'Union dans la culture
}

\section{Mireille Pongy}

\section{(2) OpenEdition \\ 1 Journals}

\section{Édition électronique}

URL : http://journals.openedition.org/conflits/385

DOI : $10.4000 /$ conflits.385

ISSN : $1777-5345$

Éditeur :

CCLS - Centre d'études sur les conflits lilberté et sécurité, L'Harmattan

\section{Édition imprimée}

Date de publication : 15 janvier 1997

ISSN : 1157-996X

Référence électronique

Mireille Pongy, «Entre modèles nationaux et eurogroupes d'intérêts professionnels : l'action de l'Union dans la culture », Cultures \& Conflits [En ligne], 28 I hiver 1997, mis en ligne le 16 mars 2006, consulté le 30 mars 2021. URL : http://journals.openedition.org/conflits/385 ; DOI : https://doi.org/10.4000/ conflits.385

Ce document a été généré automatiquement le 30 mars 2021.

Creative Commons License 


\title{
Entre modèles nationaux et eurogroupes d'intérêts professionnels : l'action de l'Union dans la culture
}

\author{
Mireille Pongy
}

\section{Mireille PONGY ${ }^{1}$}

2 Parallèlement à la forte croissance urbaine et au développement de l'Etat Providence, l'action des Etats de l'Union européenne en faveur des arts et de la culture s'est accrue. A l'image de son inscription relativement tardive sur les agendas nationaux, le domaine culturel est aujourd'hui touché, après d'autres, par l'émergence d'un nouveau lieu de ressources et d'élaboration de l'action publique. L'approche utilisée dans ce texte privilégie la manière dont les différents modèles nationaux d'action publique se confrontent et informent la construction en cours de l'action culturelle communautaire et de sa représentation. L'analyse du processus de négociation en vue de l'adoption des programmes élaborés par la Commission d'une part, la prise en compte des conditions spécifiques de l'action culturelle communautaire en termes d'énonciation de la souveraineté nationale et de vote à l'unanimité d'autre part, mettent en effet en relief le rôle des Etats membres. Il ne s'agit pas pour autant de prendre position entre " deux approches génériques ", l'une mettant l'accent sur l'action entre les Etats-Nations, l'autre sur l'émergence d'un espace d'action politique dont les composants se situent au-delà des seuls Etats ${ }^{2}$, mais de préciser l'angle de lecture choisi dans ce texte qui n'est évidemment pas indifférent quant à la nature des conclusions tirées. Par ailleurs, l'analyse d'un secteur particulier de l'action communautaire implique de prendre en compte les temporalités et capacités différenciées de l'intervention communautaire dans les différents domaines. La différence entre une politique agricole commune ou de la concurrence beaucoup plus anciennes que l'action culturelle de la Communauté reconnue officiellement par le Traité de Maastricht de 1992, pointe la difficulté de " la montée en généralité ", pour reprendre une expression de E. Friedberg, à partir d'un 
seul secteur. On s'attachera dans un premier point à décrire la position des Etats membres et des institutions communautaires, ainsi que les configurations d'alliance qui réunissent et/ou opposent ces acteurs. On analysera dans un second point la mise en place de groupes d'intérêts professionnels transnationaux au niveau communautaire et celle de nouveaux modes de médiation entre ces groupes et les institutions communautaires. Les secteurs qui composent le domaine culturel renvoient à des communautés nationales de politiques, dont les modes de gestion et de représentation, dus notamment à un mode différencié d'action publique, sont largement différents. Bien que participant d'un large mouvement de transnationalisation, les regroupements d'acteurs qui se constituent autour de l'action culturelle communautaire se superposent davantage qu'ils ne contribuent encore à la recomposition des systèmes nationaux et subnationaux. On s'attachera enfin dans une dernière partie à dégager les enjeux d'un renforcement de l'action culturelle de l'Union européenne. Institutions communautaires et logiques nationales différenciées d'élaboration de l'action culturelle communautaire L'inscription des questions culturelles sur l'agenda communautaire est récente. Une première communication de la Commission date de 1977 ; deux autres ont suivi en 1982 et 1987. Une dernière élaborée en 1992 préfigure l'article 128 du Traité de Maastricht qui consacre quasi constitutionnellement la légitimité d'une action de l'Union dans ce domaine. Le principe de subsidiarité qui inspire l'ensemble du Traité, est particulièrement réaffirmé dans l'article 128 et rappelle que la compétence culturelle relève d'abord des Etats membres. L'obligation du vote à l'unanimité, la procédure de co-décision avec le Parlement, la consultation du Comité des Régions, l'exclusion de toute action d'" harmonisation des dispositions législatives et réglementaires des Etats membres " constituent le cadre réglementaire du processus de décision de l'action culturelle. Au sein de la Commission, le domaine culturel est pris en charge par plusieurs Directions Générales (DG). La DGXVI qui gère les fonds structurels et les programmes régionaux, attribue des financements importants à des régions et à des communes rurales pour la sauvegarde et la valorisation de leur patrimoine, ainsi que pour la promotion du tourisme culturel ${ }^{3}$. Instruments principaux du renforcement de la cohésion économique et sociale au sein de l'Union, les Fonds structurels accordent cependant jusqu'à présent une place relativement modeste aux projets culturels. Compte tenu de la nature complexe, à la fois marchande et non marchande des biens culturels, d'autres directions générales parmi les plus influentes, comme celles de la concurrence (notamment sur la question des aides d'Etat aux industries culturelles nationales), des relations extérieures, ou du marché intérieur interviennent sur le domaine. C'est cependant la DGX à vocation sectorielle qui est officiellement en charge des secteurs de l'information, de l'audiovisuel, de la communication et de la culture et plus particulièrement sa direction " D ", responsable de l'action culturelle et de la politique audiovisuelle. La direction D gère plusieurs programmes : Ariane pour la traduction, Kaléidoscope pour les activités artistiques et culturelles, Raphaël pour le patrimoine, Media I et aujourd'hui Media II pour le cinéma et l'audiovisuel. Les bénéficiaires de ces programmes étant les professions culturelles, cette $D G$ est leur interlocuteur privilégié ${ }^{4}$. Elle gère un faible budget $^{5}$ et ne constitue pas une DG puissante, d'autant plus qu'elle a la charge délicate de gérer l'information et la communication de l'action de la Commission. Plutôt proche des positions françaises ${ }^{6}$, elle est, au sein de la Commission, le lieu d'énonciation d'une spécificité du domaine culturel (la célèbre exception culturelle), qui fonde la légitimité d'une protection des arts et de la culture par l'autorité politique ; dit autrement, d'un 
renforcement de sa régulation politique, contre une domination trop forte de la régulation par le marché. La DGX est chargée d'exercer le " droit de regard culturel " que l'article 128 accorde sur l'ensemble des activités communautaires et a notamment fait adopter par le Conseil une proposition prônant une meilleure intégration de la culture dans les projets de développement régional ${ }^{7}$. La procédure de co-décision permet, depuis le Traité de Maastricht, au Parlement Européen de jouer un rôle de plus en plus important dans l'élaboration des politiques ${ }^{8}$. Soucieux de préserver et de développer un espace culturel européen diversifié, ainsi que les conditions de création et de développement d'une industrie de programmes, il est très attentif à ces questions à travers les nombreux rapports qu'il leur consacre et l'activité importante de sa commission culture. Le Parlement Européen soutient quasi systématiquement les propositions de la Commission qui visent à développer l'intervention communautaire. Le sens des amendements qu'il propose est toujours celui d'une augmentation de la dotation budgétaire et de la durée des programmes. Il se heurte très souvent au Conseil et presque tous les programmes font l'objet d'une procédure de conciliation entre le Parlement Européen et le Conseil. Tant le Parlement Européen que la Commission portent globalement une logique de renforcement de l'action communautaire qui concourt à l'intégration communautaire. Cela ne signifie pas pour autant une absence de clivages en leur sein. Si la DGX a le pouvoir d'initiative dans ce domaine, elle connaît des difficultés à faire reconnaître le statut particulier des biens culturels par les autres directions générales qui considèrent que les normes du marché et de la libre concurrence doivent s'appliquer à tous les biens et services. De la même façon, l'ensemble des députés européens ne sont pas uniment favorables au renforcement de l'action culturelle communautaire. Le récent vote de la directive " Télévision sans frontières " a fait apparaitre des clivages politiques transnationaux entre partis libéraux et populaire européen d'une part, partis socialiste et vert de l'autre, mais également des clivages nationaux transcendant les clivages politiques: une partie du RPR français a déserté son camp politique traditionnel et voté aux côtés des socialistes, les amendements du PE renforçant l'impact de la directive, tandis que Forza Italia votait avec ses alliés " naturels ". Si les clivages nationaux le disputent aux clivages politiques, signe de la double logique à l'œuvre dans la mise en place d'un espace public européen à travers le Parlement, le Conseil constitue le lieu d'élection de la confrontation entre modèles nationaux d'intervention publique. Examinons d'abord le cas de la Présidence de l'Union. Bien que supposée porter l'intérêt général communautaire au même titre que la Commission ou le Parlement, la Présidence de l'Union européenne est d'abord, pour l'Etat qui l'occupe, un moment privilégié de maitrise de l'agenda où il est dans les meilleures conditions pour faire passer un certain nombre de ses priorités. Au cours du premier semestre de 1995, la présidence française a ainsi été particulièrement active ${ }^{9}$, tandis que la présidence italienne du premier semestre 1996 soutenait la DGX dans sa volonté de mieux intégrer la préoccupation culturelle dans les programmes régionaux. Ce n'est pas le cas de la plupart des Etats qui accordent en général une faible importance à ce domaine. En-deçà de la présidence, le rôle du Conseil, et en l'occurrence, des Etats membres est essentiel dans la mise (ou non) sur agenda et surtout dans la prise (ou non) de décision quant aux questions culturelles. Le critère déterminant de la position des Etats membres est leur propre modèle d'intervention publique. Chacun tente plus ou moins de l'imposer ou de le projeter au niveau européen face à l'enjeu que constitue la construction en cours d'un modèle d'intervention de l'Union dans ce domaine. Loin de se réduire au jeu des seuls 
acteurs étatiques (politiques et administratifs), ces modèles impliquent des modes de médiation entre professions culturelles et autorités politiques, entre autorités politiques de niveau différent, des positionnements variés des partis politiques... Ils expriment des représentations différentes du rôle de l'Etat et plus largement du politique, ainsi que des modes différents d'action dans le domaine de la culture. Ils informent l'action de l'Union qui ne se fonde pas sur une table rase, sur laquelle les instances proprement communautaires et en premier lieu la Commission élaboreraient l'action communautaire. Souligner ce que la construction communautaire doit à la confrontation des modèles nationaux dans un domaine où la prise de décision requiert l'unanimité, où la réaffirmation du principe de subsidiarité souligne la compétence culturelle des Etats et où la position de la DGX est souvent minoritaire - la DGX n'est pas la DGIV (concurrence) - incite à tenir compte du fait que l'action culturelle n'est pas un domaine prioritaire des instances communautaires. Si la logique de confrontation de modèles nationaux d'intervention publique semble ici plus en situation de force que dans d'autres domaines, cela ne signifie pas pour autant que la Commission et, en l'occurrence, la DG X ne soit pas en mesure de jouer un rôle d'entrepreneur politique ou d'animateur. Cependant, contrairement à d'autres secteurs, une des ressources dont dispose la Commission pour affirmer son autonomie, est ici particulièrement fragile. L'évocation de l'intérêt communautaire par la DGX ${ }^{10}$ est d'autant plus difficile que la définition de celui-ci n'est pas stabilisée et est, au contraire, l'objet de controverses entre les Etats membres. L'action culturelle s'insère dans un cadre intellectuel et normatif communautaire qui alimente les grandes orientations des actions publiques et est structuré autour des normes de marché et de subsidiarité ${ }^{11}$ inscrites dans la culture politique de certains pays et étrangères à d'autres. Une autre norme régit le domaine culturel, celle de la spécificité culturelle énoncée précédemment. Notre hypothèse est que la construction de l'intérêt communautaire et de l'action culturelle repose d'abord sur la confrontation de ces trois normes portées de façon différenciée par les Etats membres. Les négociations autour de l'adoption des programmes culturels font apparaître des clivages entre les Etats, selon que leur tradition politique est plus ou moins familière de ces normes; dit autrement, selon la nature libérale, subsidiaire ou décentraliste, interventionniste ou centraliste de leur propre modèle d'action dans le domaine. La norme du marché plaide pour un rôle " retenu " de l'instance politique et, par conséquent, de l'instance politique communautaire dans le domaine culturel qui relève d'abord de l'initiative privée, individus ou entreprises, avant que de relever de l'action publique. Certains pays sont proches de cette vision qui ne fait pas du domaine une dimension importante de l'action publique. De même, la norme du marché dominante dans la construction communautaire est peu compatible avec le développement d'une action culturelle communautaire qui signifierait une prérogative d'un lieu " central " du politique. Les pays habituellement les plus hostiles au développement de l'action culturelle communautaire sont les pays de forte tradition libérale, au premier rang desquels la Grande-Bretagne. Le principe de subsidiarité, en vigueur dans l'action culturelle en Allemagne, énonce la part fondamentale réservée à l'initiative des communes, ainsi que la compétence des Länder en la matière. Le niveau fédéral est constitutionnellement peu présent, mais il est habilité à intervenir sur des questions qui relèvent des relations extérieures ou qui sont mieux traitées à ce niveau qu'à celui des Länder, comme celle du statut social des artistes. Mais les questions culturelles sont traités prioritairement au plus près du citoyen, c'est à dire par les communes et en particulier par les villes. A l'origine du rappel du principe de 
subsidiarité dans l'article 128 , contre l'avis du gouvernement français, les Länder allemands sont vigilants à son respect par la Communauté, comme ils le sont à l'égard de la fédération en Allemagne. Tant la norme du marché que celle de la subsidiarité vont de pair avec une faible implication des échelons politiques centraux, suspectés de favoriser un art officiel ou dénoncés dans leur volonté d'instrumentaliser la culture à des fins de propagande, comme ce fut le cas lors des dictatures fascistes ou communistes au cours de ce siècle. A contrario, la norme de la spécificité culturelle va de pair avec une forte intervention de l'autorité politique, car le patrimoine, les arts ou les industries culturelles doivent être soutenus par les autorités politiques. Le pays dont l'action est la plus proche de cette norme est la France, dont la politique culturelle est particulièrement pilotée par l'échelon central de l'autorité politique. Le caractère volontariste de la politique culturelle française constitue une référence bien que controversée pour certains pays (Espagne et Italie notamment), et surtout pour les professions culturelles. D'autres facteurs, comme la disparité très forte des Etats membres quant à leur puissance/faiblesse économique, démographique, politique, jouent également un rôle non négligeable. L'arrivée récente de trois " petits pays " du " Nord " (Suède, Finlande, Autriche) a modifié l'équilibre entre Etats et introduit une clause quasi systématique mentionnant l'attention particulière accordée aux petits pays dans chaque programme. Le rééquilibrage de l'Union en faveur de ces pays renforce leur position face " aux grands ", notamment face aux projets de la France, caractérisés par leur importance et leur volonté structurante, ou contrecarre les réticences liées à la position hégémonique de la langue, que manifeste la GrandeBretagne à l'égard du développement du programme Ariane de traduction, auquel ces petits pays sont a contrario très attachés. Le rôle renforcé du Parlement Européen et l'élargissement de l'Union européenne à trois nouveaux membres ont amené une extension du nombre d'acteurs concernés, source de complexité et de rallongement accrus du processus de décision. Cette évolution amène moins un affaiblissement du pouvoir d'initiative de la DGX que son repositionnement comme entrepreneur politique ou animateur, toujours plus obligé entre les différents protagonistes, notamment entre le Conseil et le Parlement Européen. Les tribulations de la directive Télévision sans Frontières offrent une illustration du rôle incontournable de la Commission et des limites de la procédure de co-décision. Les 314 voix de majorité nécessaires pour que le Conseil modifie sa position n'ont pas été atteintes lors du vote du Parlement. Cependant, en modifiant sa proposition par l'adoption de certains amendements du Parlement Européen, la Commission a autorisé la délibération du Conseil à la majorité qualifiée et non plus à l'unanimité et joué un rôle dans le processus de décision ${ }^{12}$. Des coalitions d'alliances à géométrie variable unissent ces différents acteurs. Cependant une alliance fréquente lie, de façon différenciée selon les programmes, un pays comme la France, certains pays du Sud, la DGX et le Parlement Européen. Cette coalition cherche à développer l'action communautaire et fait prévaloir, au nom de la spécificité culturelle, la nécessité d'une logique politique de régulation du domaine, notamment celui des industries culturelles. La logique d'agrégation des acteurs est ainsi structurée autour des programmes (dotation budgétaire, durée, capacité structurante d'un milieu transnational d'opérateurs), et non pas autour de leur statut, étatique ou communautaire. Force est de constater que le renforcement de l'action culturelle communautaire est davantage porté par la position centraliste française que par la position libérale britannique ou par celle des Länder allemands, qui estiment que l'Union ne doit se préoccuper que modérément des questions culturelles. Cependant, le 
développement de l'action de l'Union ne concerne pas les seules autorités politiques et administratives nationales et communautaires mais également les intérêts professionnels. Groupes d'intérêt professionnels et modes de médiation L'intérêt relativement récent des instances communautaires pour le domaine de l'action culturelle explique que ses effets induits relèvent encore davantage de son existence même et de sa montée en puissance postulée que de la mise en œuvre de son action. Une des fonctions explicites des programmes culturels communautaires est la construction de coopérations entre acteurs nationaux autour de projets et, au-delà, la recomposition transnationale des systèmes d'acteurs. Le caractère souvent opportuniste ou volontariste des coopérations rejaillit sur leurs effets d'entraînement qui sont de ce fait encore restreints. Mais ils le sont d'autant plus que les programmes relèvent davantage d'une logique de saupoudrage que d'une action forte et cohérente. L'inscription de l'article 128 dans le Traité de Maastricht avait pourtant pour objectif de dépasser le stade des actions ponctuelles et expérimentales au profit de programmes plus cohérents et pluriannuels. La réticence persistante de certains Etats membres à doter ceux-ci d'une durée et d'une dotation budgétaire suffisante est en grande partie responsable de leurs difficultés à structurer un milieu transnational d'artistes et d'opérateurs culturels. En revanche, la montée en puissance de l'UE provoque, dans une sorte de mouvement " bottom-up ", à la fois une certaine recomposition des systèmes d'acteurs au niveau national et surtout leur élargissement. La mise en place en Allemagne, au début des années 1980, d'une organisation nationale d'harmonisation et de représentation des intérêts de l'ensemble des professions culturelles (le Kulturrat) procède certes de la prise de conscience du poids non négligeable du Bund en dépit de ses faibles compétences dans le domaine, mais tout autant de la nécessité ressentie par les professions de présenter " un front uni " allemand face à Bruxelles ${ }^{13}$. Cependant, le nouvel intérêt communautaire pour le domaine culturel amène surtout la création d'une " couche " supplémentaire d'acteurs qui se superpose aux acteurs nationaux. Le nombre élevé des " eurogroupes " d'intérêt professionnel est quelque peu paradoxal, au regard du niveau de ressources que l'Union alloue actuellement aux divers secteurs culturels et ne se comprend que par l'attitude d'anticipation qui les animent: anticipation autant sur l'accroissement du niveau de ressources financières que sur les futures capacités réglementaires de l'Union. La mise en place de regroupements professionnels est antérieure à l'inclusion de l'article 128 dans le Traité de Maastricht, mais celui-ci a contribué à précipiter le mouvement. Le développement important de structures européennes de représentation des intérêts sociaux des secteurs culturels se superpose ainsi aux modes internationaux de représentation dont s'étaient déjà dotées certaines professions, notamment celles du patrimoine (musées, bibliothèques). La multiplicité actuelle de ces groupes recouvre à la fois des secteurs (spectacle vivant, cinéma), des professions (artistes, opérateurs culturels, formateurs, administrateurs), ainsi que des institutions (théâtres, festivals, centres culturels indépendants, centres de formation). Dans les secteurs du spectacle vivant et du cinéma, plus particulièrement pris en compte ici, ces groupes se donnent de façon plus ou moins importante, mais non exclusive, des fonctions qui vont de celles de " lobby " ou groupe de pression, à celles de socialisation mutuelle de leurs membres à travers l'échange d'informations, d'expériences et de savoir-faire, en passant par la production de réflexions destinées aux institutions communautaires. Dit autrement, les activités de ces groupes se situent sur une ligne qui relierait l'arène, " espace de négociation " de l'action publique, au forum, " espace de débat ", l'un et l'autre étant, en l'état actuel d'ouverture et de 
plasticité de la construction de l'action culturelle européenne, fortement mêlés ${ }^{14}$. Les structures les plus larges et informelles se donnent d'abord pour mission d'être des lieux de rencontre et de socialisation mutuelle. Leurs ambitions en matière d'influence sur les processus de décision ne sont pas ici essentielles, même si elles ne sont pas absentes, au moins pour l'avenir. Pour ce type de regroupement, il importe de pourvoir leurs adhérents en informations susceptibles de contribuer à leur meilleure compréhension d'un dispositif d'aides complexe, qui leur est étranger par son originalité. La production non négligeable de guides, vade-mecum et autres annuaires entend fournir aux professions le mode d'emploi des soutiens communautaires à la culture et constitue un premier outil d'acculturation au dispositif institutionnel communautaire. La vision européenne de ces groupes est dans une large mesure celle d'usagers de ce dispositif. D'autres groupes développent principalement une fonction de forum où s'effectue un travail de réflexion et d'harmonisation des représentations de leurs membres dans le domaine de la culture. Le débouché de ce travail, qui se veut constructif, est la rédaction de textes diffusant idées et réflexions auprès des instances communautaires, suggérant par exemple des modes d'optimalisation de leurs fonctions et réagissant plus précisément aux propositions de la Commission. Certaines organisations endossent enfin explicitement le rôle de groupe de pression défendant d'abord leurs propres intérêts. Plus actifs et plus puissants dans les industries culturelles et notamment dans le cinéma et l'audiovisuel que dans les secteurs dépendant quasi-exclusivement des fonds publics, ils tentent d'intervenir plus précocement et d'influer en amont la formulation même des textes. C'est ainsi qu'un groupe de pression français est parfois informé des premières versions des propositions de la Commission, avant même le Centre National de la Cinématographie (CNC), chargé de représenter et de défendre la position française au niveau des instances communautaires ${ }^{15}$. Cette typologie succincte ne doit pas occulter le fait dominant que sont la multiplicité, la fragmentation, l'instabilité, l'ouverture de ces groupes, dues notamment à la faiblesse de leurs ressources et à la fragilité de leur représentativité. Cette situation accrédite l'idée de la mise en place au niveau européen d'un modèle pluraliste anglo-saxon de représentation des intérêts et de médiation entre les groupes d'intérêt et l'administration communautaire. Cette idée n'est pas sans lien avec l'activité importante des Britanniques dans la constitution d'une scène culturelle communautaire ${ }^{16}$. La mobilisation différenciée des nationalités s'explique essentiellement par l'importance et les modes d'action publique dans le champ culturel national, et donc, du niveau et du type de ressources dont disposent les professions dans les Etats membres. A cet égard, la moindre mobilisation des professions françaises ou allemandes semble liée à la situation relativement privilégiée dont elles bénéficient au niveau national. Les Britanniques sont également plus familiers de la pratique du lobbying, mode de médiation développé majoritairement autour des institutions européennes. De même, l'élaboration d'un langage communautaire se fait clairement sous l'influence de la langue anglaise, et les mécanismes d'acculturation des professions culturelles sont largement développés ${ }^{17}$. Les termes de réseau (network), lobbying, advocacy group, ou encore forum font partie intégrante du vocabulaire des professions. Celui de réseau est d'autant plus fréquemment utilisé par les professions, pour définir leurs regroupements, qu'il est valorisé pour ses qualités énoncées de fluidité, d'adaptabilité; pour sa capacité supposée à favoriser les échanges d'information, d'expériences et de savoir-faire, et à utiliser les nouveaux outils technologiques de communication.... " Le réseau " défini normativement et pratiqué par les acteurs 
culturels apparaît comme une forme post-nationale du groupe d'intérêts fortement influencée par les modes anglo-saxons de représentation. Cette acception se distingue du sens que lui accordent les politistes, en tant qu'" outil descriptif ", non pas en l'occurrence d'un " Etat fragmenté ", pour reprendre les termes de P. Le Galès ${ }^{18}$, mais d'une instance politique polycentrique en voie de constitution. Le fait que " l'étude des réseaux d'action publique s'enracine dans une conception des politiques publiques qui souligne leur nature incrémentale " prédispose ce concept à être un outil particulièrement performant de l'analyse de l'action publique communautaire, dont un des caractères essentiels est a priori son pragmatisme à travers notamment les effets récurrents de " spill over ". Entendu comme " meso-level concept " politologique, le réseau s'articule autour de la notion d'action publique communautaire, sous la forme concrète de programmes. Les groupes d'intérêts professionnels (définis comme réseaux d'acteurs par leurs protagonistes) sont donc partie prenante, à des degrés divers, des réseaux qui se créent autour de l'action communautaire et participent à leur construction à côté des administrations nationales et communautaires. Entendus comme " le résultat de la coopération plus ou moins stable, non hiérarchique, entre des organisations qui se connaissent et se reconnaissent, négocient, échangent des ressources et peuvent partager des normes et des intérêts... dans un environnement complexe "19, les réseaux de l'action culturelle communautaire sont encore instables, compte tenu de la jeunesse de l'action culturelle communautaire. Ils le sont d'autant plus que l'on prend en compte la dimension interpersonnelle, la rotation des individus étant importante à Bruxelles ${ }^{20}$. Ces différents éléments d'analyse plaident pour la construction d'un mode de relations entre professions et autorités politicoadministratives marqué par la fragmentation des groupes d'intérêts, qui n'exclut cependant pas la mise en place de réseaux d'action culturelle et les prémisses de formes plus institutionnalisées de concertation et de négociation. Il est en effet difficile de dire si l'actuel modèle pluraliste se stabilisera. Dans la mesure où le défi de la construction européenne se formule moins en termes de transposition d'un modèle national qu'en termes d'une construction originale empruntant à plusieurs modèles, il convient de noter que d'autres modèles de médiation entre intérêts sociaux et autorités politiques et administratives sont " disponibles sur le marché ". On peut en effet se poser la question des conditions d'un transfert du modèle de corporatisme sectoriel ${ }^{21}$ qui prévaut dans certains secteurs de la politique culturelle française. Ce modèle, à l'œuvre depuis une quinzaine d'années dans certains secteurs du spectacle vivant ${ }^{22}$ ou depuis plus longtemps à travers le type de concertation permanente qui préside aux relations entre le milieu professionnel cinématographique et le $\mathrm{CNC}$, ne semble pas - encore ? avoir été mis en cause par la construction européenne ${ }^{23}$. Les eurogroupes professionnels sont sensibles à l'action d'un ministère dont la visibilité et les moyens sont supérieurs à ceux de ses homologues européens et dont le champ d'action est en France quasi-exclusivement celui de la culture professionnelle. Ayant de ce fait une relation privilégiée avec les professions, sa position énoncée de défenseur des artistes lui vaut le soutien fréquent des professions au niveau communautaire. L'exemple d'une organisation qualifiée par ses responsables d'umbrella organization offre une autre occasion de s'interroger sur les contours futurs des relations entre groupes professionnels et instances communautaires. Récemment créé, le Forum européen pour les arts et le patrimoine regroupe plusieurs secteurs (musique, théâtre, danse, patrimoine), et mène un travail d'harmonisation et de représentation de l'ensemble des intérêts professionnels. Si ce regroupement ou d'autres se développaient, ils pourraient 
constituer les prémisses d'une forme moins sectorielle et plus centralisée de négociation des intérêts, rapprochant la scène européenne de la situation allemande qui prévaut tant au niveau des Länder qu'au niveau fédéral avec la Dachverband qu'est le Kulturrat allemand. Dans cette perspective, la lutte des groupes pour leur positionnement comme interlocuteur reconnu par les instances communautaires est vive. La recommandation récente, adressée aux adhérents nationaux de l'Informal European Theatre Meeting, de faire connaitre et reconnaitre le travail de celui-ci auprès de leurs représentants nationaux, membres du Parlement Européen ou fonctionnaires ministériels et conseillers divers, en est un signe. Le financement d'une partie de leurs activités de fonctionnement vient d'être accordé à quelques groupes, sous certaines conditions (représentativité, qualité de la réflexion, dynamisme), dans le cadre du programme Kaléidoscope 2000. Considéré par les groupes comme une étape essentielle à leur reconnaissance comme partenaire de l'élaboration et de la négociation de l'action, ce financement contribue à la structuration des réseaux de l'action culturelle. La contribution de la DGX à leur institutionnalisation n'est d'ailleurs pas seulement profitable aux eurogroupes, mais aussi à elle-même. Si sa pratique de la consultation des groupes d'intérêts est relativement récente par rapport à celle des autres DG, elle a aujourd'hui, à l'instar de ses homologues, de plus en plus besoin de ces interlocuteurs, pour affermir sa position à la fois au sein de la Commission et par rapport aux Etats membres ${ }^{24}$. Le développement de l'action de l'Union et la problématisation d'une " identité culturelle européenne " Certains auteurs soulignent le paradoxe qui réside dans l'affirmation combinée de la norme néo-libérale et de la nécessité de renforcer les politiques communes ${ }^{25}$, ou dans l'extension du vote à la majorité et l'approfondissement de l'action communautaire dans une perspective néolibérale ${ }^{26}$. Pour pertinent et séduisant que soit ce paradoxe, il importe de le déconstruire et de l'affiner pour mettre à plat les contradictions à l'œuvre dans la construction de l'action culturelle. L'analyse des processus de mise sur agenda et de prise de décision dans ce domaine fait apparaître que les débats en cours portent à des degrés divers et de différentes façons sur le renforcement de l'intervention de l'Union par la mise en place des conditions d'une meilleure efficacité du processus de décision, au premier rang desquelles la transformation du vote à l'unanimité en vote à la majorité qualifiée. Cette question est d'autant plus importante que l'enjeu financier, comme pour les autres domaines, sauf ceux des politiques agricoles et régionales, est relativement négligeable - au moins pour les grands pays -, comparé aux ressources dont disposent les secteurs aux niveaux nationaux. En regard, le coût de négociations longues nécessaires à la formation $d u$ consensus, favorisant la pratique du marchandage et aboutissant généralement au dégagement du plus petit dénominateur commun, requises par le vote à l'unanimité, est considérable. C'est dans cette perspective, qu'un nombre important d'acteurs (Etats et groupes d'intérêts) ont demandé que la Conférence Intergouvernementale se prononce dans ce sens. Pour autant, la mise en place de cette nouvelle procédure laisse entière l'incertitude quant au sens dans lequel ira ce renforcement. Le vote à la majorité qualifiée aiguise les contradictions entre les parties en présence et permet une plus grande efficacité de coalitions déjà existantes ${ }^{27}$ mais ne prend pas position, en tant que tel, sur les logiques à l'œuvre; tant la norme du marché que celle de la subsidiarité ou encore celle du centralisme politique sont compatibles avec ce vote. La difficulté de la mise en place de nouveaux programmes après Maastricht tient essentiellement à l'hostilité de certains Etats membres. Les négociations longues et âpres qui ont marqué l'adoption récente 
des programmes Kaléidoscope et MEDIA II, en sont le témoin. La proposition faite par la DGX de mieux doter ces programmes et d'allonger leur durée avait reçu l'appui du gouvernement français et des professions concernées. L'hostilité des représentants des gouvernements britannique, allemand et hollandais, le faible intérêt accordé à ce domaine par d'autres, la clause du " petit pays " ont fait échec aux propositions de la Commission. En n'augmentant ni la dotation budgétaire, ni la durée des programmes, le Conseil s'est privé d'accroître la capacité structurante des programmes sur le système d'acteurs $^{28}$. Les débats qui ont donné lieu à la reconduction récente de la directive Télévision sans Frontières (et des quotas) posent le problème du renforcement des capacités de réglementation de l'Union. Ce dossier a vu s'affronter deux positions, dont l'une prônait un renforcement de la directive, et l'autre sa suppression ${ }^{29}$. Autour de la première position s'agrégeaient les autorités françaises, les professions cinématographiques, en particulier françaises, soutenues par l'ensemble des professions culturelles, le Parlement européen et à l'origine la DGX ; la seconde était portée par la Grande-Bretagne suivie par la plupart des autres Etats membres. La situation de l'industrie cinématographique est sensiblement différente en France et dans les autres pays européens. L'existence d'une industrie cinématographique française et donc de professions est redevable de l'ensemble des mécanismes de soutien mis en place par l'Etat, via le CNC depuis 1946. Si cet organisme gère l'attribution des différentes formes de soutien, il organise surtout la concertation permanente des autorités politiques avec l'ensemble des professions du secteur. Cette configuration spécifique en Europe du soutien de l'Etat à une industrie culturelle explique à la fois le haut degré d'intégration de la politique cinématographique française et le fait que professions et autorités politiques et administratives nationales réagissent de façon largement concertée aux propositions et actions communautaires ${ }^{30}$. Dans les autres pays européens, les industries cinématographiques ont été en grande partie laminées par la concurrence de la télévision et par l'hégémonie des produits américains diffusés sur les écrans européens. Ayant intérêt à préserver les conditions du développement de sa propre industrie, " l'entreprise France " (administration nationale et professions) porte le dossier au niveau communautaire et développe, pour ce faire, l'argument de la spécificité culturelle. Conscientes des menaces que font peser sur le modèle français de soutien public à la production cinématographique, l'ouverture du marché européen aux industries américaines et la politique de déréglementation et de remise en cause des aides accordés par les Etats à leur industrie cinématographique nationale, les professions françaises ont créé des groupes de pression puissants et surtout vigilants. Ils ont joué un rôle important lors des négociations du GATT de décembre 1993. Très présents dans les négociations permanentes qui se mènent dans le cadre de l'Organisation Mondiale du Commerce, ils ne cessent de dénoncer les négociations bilatérales qui s'y déroulent et l'absence d'une position européenne. Les demandes adressées à l'Union par les professions cinématographiques ne concernent que marginalement le montant des soutiens accordés par le plan MEDIA II ${ }^{31}$ mais bien plus les procédures de réglementation visant à favoriser le développement et/ou la renaissance d'une industrie cinématographique européenne ${ }^{32}$, dans des conditions régulées de la concurrence internationale, notamment américaine. Sur ce point, les professions cinématographiques reçoivent le soutien de l'ensemble des professions culturelles. Un de leurs plus importants groupes d'intérêts a adressé à la Conférence intergouvernementale une demande de nouvelle rédaction de l'article 128 , dans laquelle serait clairement reconnu le droit des Etats d'aider leurs industries 
cinématographiques nationales. Dans le conflit qui oppose les professions cinématographiques aux majors américaines, les premières mettent en avant la préservation d'un espace européen de production et de diffusion. La construction d'un espace aujourd'hui potentiel, sur la base des espaces nationaux et des coopérations transnationales existantes, est essentielle à la survie de ces professions et des industries cinématographiques européennes. La reconnaissance d'une régulation politique des conditions de la concurrence n'étant pas reconnue par la plupart des Etats membres et par les directions générales les plus influentes de la Commission, il devient impératif pour les acteurs concernés d'ancrer ce type de revendications sur une autre norme, celle de la spécificité culturelle. Entendue ici comme ressource stratégique, celle-ci est fondée sur la problématisation d'une représentation européenne de l'art et de la culture, dans laquelle les notions d'œuvre, d'auteur et d'artiste sont centrales. Cette construction s'oppose à la représentation d'une culture " américaine " incarnée par les majors hollywoodiennes dans laquelle la figure du producteur est essentielle. Le conflit entre droits d'auteurs et copyright est emblématique de cette opposition. Plus que le renforcement de l'action communautaire dans le secteur culturel, c'est la reconnaissance de la légitimité de cette spécificité culturelle européenne qui constitue la ligne directrice des propositions des professions quant à une meilleure application de l'article 128 et à sa nouvelle rédaction. Au-delà du secteur cinématographique, la reconnaissance d'une spécificité des biens et de l'action culturelle constitue le référent commun des professions et est la condition nécessaire sinon suffisante, de la création d'un espace européen dans lequel la construction des règles serait co-produite par les représentants de ces professions. En prenant ses distances avec des finalités économiques, cette construction s'inscrit dans une logique politique de régulation, que l'intervention publique soit de nature plutôt redistributive (spectacle vivant), ou règlementaire (cinéma). L'élaboration de l'action culturelle communautaire paraît aujourd'hui soumise à la construction d'une représentation commune de l'action dans ce domaine. Cette phase met en relief la confrontation entre modèles nationaux différents d'intervention publique dans le domaine culturel et explique la difficile énonciation d'un intérêt communautaire par la Commission. Structurés de façon plus ou moins exclusive autour des normes de marché, de subsidiarité et de centralisme politique, ces modèles informent l'élaboration de l'action culturelle communautaire. Pour légitimer leur propre position, les instances communautaires que sont la Commission et le Parlement Européen ont tendance à s'appuyer davantage sur cette dernière norme qui fonde un renforcement de l'action de l'Union. Au-delà d'une reconnaissance et d'un soutien à la diversité des cultures européennes, c'est la question d'une problématisation de l'identité culturelle européenne, fut-elle déclinée en termes de pluralités, qui est posée. C'est bien dans cette capacité de problématisation que se joue le " plus " apporté par le niveau communautaire à l'action des Etats membres et la spécificité à venir de l'autonomie des instances communautaires, et en premier lieu de la Commission, par rapport aux Etats membres. Les industries culturelles posent des problèmes différents des secteurs traditionnellement plus dépendants des financements publics, comme le spectacle vivant ou le patrimoine. Le spectacle vivant renvoie essentiellement à la structuration de coopérations et d'un milieu transnational d'opérateurs, par ailleurs lentement en cours. Le traitement de la question patrimoniale et de l'histoire, requiert en plus de cette synergie programmée, une recherche et une écriture de l'identité européenne que l'Union a chargé certains historiens de tenter. Le caractère domestique ou national de ces secteurs s'accomode 
relativement bien d'une action subsidiaire de la Communauté. Mais ce sont surtout les industries culturelles et en particulier cinématographiques, dont le marché est mondialisé, qui posent la question d'une intervention spécifique plus forte de l'Union face à des concurrences extérieures et remettent en cause l'hégémonie des normes du marché et de la subsidiarité dans la construction européenne. L'appartenance des politiques culturelles aux catégories des politiques du Welfare, des politiques industrielles, mais aussi d'une certaine façon à celle des high policies auxquelles est fortement attachée la notion de souveraineté nationale, pose des problèmes particulièrement complexes de modes d'action.

\section{NOTES}

1. Chargée de recherche au CERAT/CNRS.

2. Voir l'introduction de Chr. Lequesne et de $\mathrm{A}$. Smith à ce présent numéro.

3. On estime que de 1989 à 1993 plus de 400 millions d'écus ont été consacrés à des projets liés à la culture par les Fonds structurels.

4. La DGX gère également des opérations dont les destinataires sont des institutions politiques mais elles ont un caractère plus ponctuel et symbolique, comme le mois européen de la culture organisé par un Etat membre ou la capitale européenne de la culture qui échoit chaque année à une grande ville. Nous intéressant ici aux interactions entre action communautaire et professions culturelles, nous ne les prendrons pas en compte.

5. La DGX gère moins du millième du budget communautaire et la moitié des fonds destinés à des opérations culturelles et gérés par la DGXVI.

6. C'est actuellement un Français qui occupe le poste de responsable de la direction D de cette DG, dirigée par une femme luxembourgeoise.

7. De la même façon, la DGXI a la charge d'inclure les préoccupations écologiques dans les autres domaines sectoriels. Cf. Cini M., " La Commission européenne lieu d'émergence de cultures administratives, L'exemple de la DGIV et de la DGXI ", Revue Française de Science Politique, vol. 46, n 3, 1996, p. 469.

8. Earnshaw D., Judge D., " Early days : the European Parliament, co-decision and the European Union legislative process post-Maastricht ", Journal of European Public Policy, vol. 2, 4, 1995, pp. 624-49.

9. Recentrage et développement du programme MEDIA II, renégociation de la directive TSF, simplification des procédures et dotation supérieure des autres programmes, affinement d'une stratégie européenne en matière de production et de distribution des produits multimédia dans le domaine du patrimoine.

10. Pour Chr. Lequesne, les quatre ressources de l'entrepreneur politique qu'est la Commission sont le pouvoir constitutionnel d'initier l'action publique, l'évocation de l'intérêt communautaire, une aptitude à mobiliser des idées et le recours à la régulation comme mode d'action publique. Cf. Lequesne Chr., " La Commission européenne entre autonomie et dépendance ", Revue Française de Science Politique, vol. 46, n³, 1996. 
11. Muller P., " La mutation des politiques publiques européennes ", Pouvoirs, $\mathrm{n}^{\circ} 69$, 1993.

12. On temporisera donc les doutes émis par J.F. Polo sur l'autonomie du statut d'entrepreneur politique de la Commission. Polo J. F., "La Commission comme espace de négociation : le cas de la politique européenne de l'audiovisuel ", 8ème Colloque International de la Revue Politiques et Management Public, Paris, 1996.

13. Pongy M., Saez G., Politiques culturelles et régions en Europe, Paris, L'Harmattan, Collections Logiques Politiques, 1994.

14. Nous nous situons ici dans ce que B. Jobert définit comme " les approches les plus courantes des politiques publiques ". Contrairement à son analyse, il nous semble que l'ouverture ou plutôt la constitution en cours des réseaux d'action culturelle au niveau européen plaide non pas pour " une coïncidence " des espaces de négociation et de débat, mais pour leur interpénétration forte. Par ailleurs, la distinction nette de ces espaces postule une production exclusive des connaissances et des idées par la catégorie générique des " experts ", qui ne nous semble pas pertinente pour l'objet étudié ici. La confrontation permanente des groupes d'intérêts professionnels aux autorités politiques et administratives qui relève de la notion d'arène est, selon nous, productrice de connaissances nouvelles par les groupes d'intérêts eux-mêmes. Jobert B., " Rhétorique politique, controverses scientifiques et construction des normes institutionnelles : esquisse d'un parcours de recherche ", Faure A., Pollet G., Warin Ph (dir.), La construction du sens dans les politiques publiques, Débats autour de la notion de référentiel, Paris, L'Harmattan, Coll.Logiques Politiques, 1995.

15. Cette constatation rejoint des observations faites dans d'autres secteurs. Mazey S., Richardson J., " De la liberté de mœurs politiques à un style européen de politique publique ", Meny Y., Muller P., Quermonne J.L. (dir.), Politiques publiques en Europe, Paris, L'Harmattan, Coll. Logiques politiques, 1995, p. 106.

16. C'est un Britannique qui est à l'origine du groupe de pression aujourd'hui le plus puissant dans le domaine des arts et du patrimoine.

17. Sur le " jargon " bruxellois et ses usages dans les Etats membres, voir Abelès M., Bellier I., " La Commission européenne : du compromis culturel à la culture politique du compromis ", Revue Française de Science Politique, vol. 46, n³, juin 1996, p. 441.

18. Le Galès P., Thatcher M. (dir.), Les réseaux de politique publique, Débats autour des policy networks, Paris, L'Harmattan, Coll. Logiques politiques, 1995.

19. Le Galès P., Thatcher M. (dir.), op. cit., p.14. Sur l'approche en termes de réseaux, voir également Smith A., " Les idées en action : le référentiel, sa mobilisation et la notion de policy network " in Faure A., Pollet G., Warin Ph., op. cit..

20. La forte rotation des fonctionnaires nationaux au niveau européen - le passage par Bruxelles ne constituant qu'une étape dans leur carrière -, leur renouvellement notamment lors de la fin de chaque présidence, ne favorisent pas la stabilisation de ces réseaux. La définition que $P$. Le Galès donne des réseaux ne retient pas la dimension interpersonnelle. Cf. Le Galès P., Thatcher M., op. cit., p.14, note de bas de page.

21. Jobert B., Muller P., L'Etat en action, politiques publiques et corporatismes, Paris, PUF, 1987.

22. Ducrey C., Urfalino Ph., " Analyse comparative de deux directions du ministère de la Culture en France ", XIIème congrès mondial de sociologie, ISA, Madrid 9/13 juillet, 1990.

23. Dans une analyse plus large de l'évolution des modes de médiation entre administration nationale et groupes d'intérêts, Chr. Lequesne constate la tendance 
inverse. Cf. Lequesne Chr., " L'administration centrale de la France et le système politique européen " in Meny Y., Muller P., Quermonne J.L (dir.), Politiques publiques en Europe, op. cit., p.143.

24. Sidjanski D., " Nouvelles tendances des groupes de pression dans l'Union Européenne ", Meny Y., Muller P., Quermonne J.L (dir.), op. cit., p.77 ; Mazey S., Richardson J., op. cit., p. 101.

25. Majone G. D., " Déréglementation ou re-réglementation? La conduite des politiques publiques dans la Communauté européenne depuis l'Acte unique " in Jobert B (dir.)., Le tournant néo-libéral en Europe, L'Harmattan, 1994.

26. Moravcsik A., " Negotiating the Single European Act " in Kehohane R. O., Hoffmann S. (eds), The new European Community, Decision-Making and Institutionnal Change, Boulder, Westview Press, 1991.

27. Cf. Lequesne Chr., Paris-Bruxelles, Comment se fait la politique européenne de la France, Paris, Presses de la FNSP, 1993, p. 29.

28. Les représentants français ont cependant réussi à arracher une expérimentation sur deux ans, à partir de laquelle ils espèrent pouvoir renégocier une reformulation du programme en fonction des résultats de la dite expérimentation.

29. La question de l'efficacité réelle de l'application d'une telle norme alors que se développent des réseaux mondiaux d'information n'est pas prise en compte ici.

30. Pour exceptionnelle que paraisse la situation française, la plupart des gouvernements européens agissent de façon générale comme les agents des intérêts nationaux dont ils sont commanditaires (Cf. Mazey S., Richardson J., op. cit., p.104). Il semble ainsi que la construction européenne mette un temps entre parenthèses ce que $\mathrm{Ph}$. Schmitter appelle " la querelle entre Etats nationaux et secteurs industriels ou de services définis de façon fonctionnelle " qu'il dit être par ailleurs sans solution, dans certains secteurs culturels au moins. Schmitter Ph., " Quelques alternatives pour le futur système politique européen et leurs implications pour les politiques publiques européennes ", Meny Y., Muller P., Quermonne J. L. (dir.), op. cit., p. 46.

31. A titre d'exemple, le budget annuel du CNC est équivalent à celui du plan MEDIA II réparti sur cinq ans pour quinze pays.

32. Etant entendu que la renaissance d'une industrie cinématographique dans les autres pays européens dépend d'abord et avant tout de la volonté des acteurs privés de réinvestir dans ce secteur. A ce sujet, certains éléments permettent de penser que la situation évolue. L'investissement récent de Canal + en Espagne a permis de produire en 1995 une quarantaine de films, et la société Pathé commence de s'intéresser au marché anglais qui se développe à nouveau grâce aux nouveaux financements issus de la loterie nationale. A l'occasion du dernier festival de Berlin, la chaîne publique de télévision allemande ZDF vient d'annoncer son intention de consacrer des financements importants à la production d'oeuvres cinématographiques variées. 


\section{INDEX}

Index géographique : Bruxelles, Europe, Union européenne

Mots-clés : bureaucraties/ théorie des organisations, construction européenne, politiques publiques, culture(s) 